\section{Heterogeneity, consistency and model fit should be assessed in Bayesian network meta-analysis}

We read with deep interest the article by Wang et al ${ }^{1}$ aimed to compare the efficacy of 20 non-steroidal anti-inflammatory drugs (NSAIDs) in the short-term treatment of ankylosing spondylitis (AS). This Bayesian network meta-analysis indicated that etoricoxib was more effective in reducing pain in AS than some other NSAIDs. The result was very useful in the decisionmaking process, especially for pain relief in treating patients with AS. However, there are some worthwhile issues that need to be explored.

First, it is commonly agreed that meta-analysis, including network meta-analysis, should assess heterogeneity, which may be defined as the presence of variation in true effect sizes underlying the different studies. Unfortunately, however, heterogeneity was not assessed in this network meta-analysis. Between-study heterogeneity indicates the presence of effect modifiers, and variability in relative treatment effects has a bad impact on the external validity of trial evidence, and limits the ability to generalise from the results. ${ }^{2}$ There may exist some potential covariates, especially for the various drug doses and treatment durations of the same NSAID, meaning that it is highly likely that there was heterogeneity. In addition, some extremely important covariates, such as drug dose, for the same NSAID should be considered in this network meta-analysis. The authors should consider the relative contribution of true variability and random variation due to biases. Perhaps Bayesian meta-regression should be conducted to consider the covariates. Second, unlike classical meta-analysis, Bayesian network meta-analysis combines all available direct and indirect evidences on the relative treatment effects. ${ }^{3}$ Inconsistency can also be caused by the effect modifiers and specifically by an imbalance in the distribution of effect modifiers in the direct and indirect evidence. ${ }^{4}$ Consistency assessing is also worthy of expectation. Third, a better model fit can reduce the inconsistency between direct and indirect evidences. ${ }^{5}$ Thus, model fit should be assessed to assure the reliability of the results as well. In order to provide reliable evidence, a checklist, ${ }^{6}$ which is intended for those who review evidence syntheses and those submitting syntheses, should be recommended.

Above all, perhaps, heterogeneity, consistency and model fit should be assessed in this Bayesian network meta-analysis to make the results more reliable for guiding clinical practice. We respect the great contributions of the authors, and we also very much look forward to seeing authors' response to these issues.

\section{Jie Wei, ${ }^{1,2}$ Chao Zeng, ${ }^{3}$ Guang-hua Lei ${ }^{3}$}

${ }^{1}$ Health Management Center, Xiangya Hospital, Central South University, Changsha, Hunan Province, China

${ }^{2}$ Department of Epidemiology and Health Statistics, School of Public, Health, Central South University, Changsha, Hunan Province, China

${ }^{3}$ Department of Orthopaedics, Xiangya Hospital, Central South University, Changsha, Hunan Province, China

Correspondence to Dr Guang-hua Lei, Department of Orthopaedics, Xiangya Hospital, Central South University, Xiangya Road, Changsha, Hunan Province 410008, China; Igh9640@sina.cn

Acknowledgements The authors thank Tony Ades, Sofia Dias, Nicky Welton, Debbi Caldwell (School of Social and Community Medicine, University of Bristol) and Kieth Abrams, Alex Sutton, Nicola Cooper (Department of Health Sciences, University of Leicester, University of Leicester) for their course 'Indirect and Mixed Treatment Comparisons, 2015.'

Contributors JW: concept, writing; CZ: writing; Gh-L: concept, revising.

Competing interests None declared.

Provenance and peer review Not commissioned; internally peer reviewed.

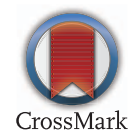

To cite Wei J, Zeng C, Lei G-hua. Ann Rheum Dis 2016;75:e5.

Received 25 September 2015

Accepted 26 September 2015

Published Online First 15 October 2015

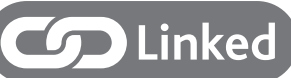

http://dx.doi.org/10.1136/annrheumdis-2015-208670

Ann Rheum Dis 2016;75:e5. doi:10.1136/annrheumdis-2015-208658

\section{REFERENCES}

1 Wang R, Dasgupata A, Ward MM. Comparative efficacy of non-steroidal anti-inflammatory drugs in ankylosing spondylitis: a Bayesian network meta -analysis of clinical trials. Ann Rheum Dis 2015; Published Online First 6 Aug 2015. doi.org/10.1136/annrheumdis-2015-207677

2 Dias S, Sutton AJ, Welton NJ, et al. Evidence synthesis for decision making 3: heterogeneity-subgroups, meta-regression, bias, and bias-adjustment. Med Decis Making 2013;33:618-40.

3 Zeng C, Wei J, Li H, et al. Comparison between $200 \mathrm{mg}$ QD and $100 \mathrm{mg}$ BID oral celecoxib in the treatment of knee or hip osteoarthritis. Sci Rep 2015;5:10593.

4 Dias S, Welton NJ, Sutton AJ, et al. Evidence synthesis for decision making 4: inconsistency in networks of evidence based on randomized controlled trials. Med Decis Making 2013;33:641-56.

5 Spiegelhalter DJ, Best NG, Carlin BP, et al. Bayesian measures of model complexity and fit. J R Statist Soc B 2002;64:583-639.

6 Ades AE, Caldwell DM, Reken S, et al. Evidence synthesis for decision making 7 : a reviewer's checklist. Med Decis Making 2013;33:679-91. 